\title{
Percepção Discente sobre o Ambiente Educacional da Disciplina de Semiologia Médica
}

\section{Student's Perception of the Educational Environment within the Medical Semiology Subject}

\author{
Sílvia Cristina Marques Nunes Pricinote ${ }^{\mathrm{I}}$ (ID \\ Artur Lorenzo Sena Gomes ${ }^{1}$ (D) \\ Alberto Monteiro Filho ${ }^{\mathrm{I}}$ (i) \\ Bruno Leonardo Wadson Silva ${ }^{\mathrm{I}}$ (D) \\ Reinaldo Elias de Souza Junior ${ }^{\mathrm{I}}$ (D) \\ Denise Milioli Ferreira ${ }^{1}$ (i) \\ Maria do Rosário Ferraz Roberti ${ }^{1}$ iD \\ Marcos Rassi Fernandes ${ }^{\mathrm{I}}$ (i)
}

\section{PALAVRAS-CHAVE}

- Avaliação Educacional.

- Estudantes de Medicina.

- Educação Médica.

- Ensino.

- Instituições de Ensino Superior.

\section{RESUMO}

Introdução: A Faculdade de Medicina da Universidade Federal de Goiás (FM-UFG) iniciou um novo processo de mudança curricular em 2014. As disciplinas de Semiologia I e II passaram a adotar metodologias ativas de ensino para adequação às necessidades da reforma curricular. O objetivo deste artigo foi avaliar a percepção dos discentes do primeiro ano da FM-UFG sobre o ambiente educacional das disciplinas de Semiologia I e II. Método: Trata-se de um estudo descritivo, do tipo corte transversal, envolvendo 86 alunos. O instrumento de coleta de dados foi o questionário Dundee Ready Education Environment Measure (Dreem), versão em português. Os resultados foram considerados em três níveis: questões individuais, cinco dimensões e Dreem global. Calcularamse a média, o desvio padrão e os respectivos intervalos de confiança de $95 \%$. Obteve-se a confiabilidade interna do Dreem pelo cálculo do alfa de Cronbach, avaliou-se a distribuição dos dados da amostra pelo teste de Shapiro-Wilk e realizou-se a comparação entre os percentuais da média dos escores dos domínios e do DRREM global pela ANOVA, seguida do teste de Tukey. Resultados: A média do Dreem global foi de 134,83/200 \pm 17,42, uma percepção mais positiva que negativa. As cinco dimensões tiveram os seguintes resultados: percepção da aprendizagem (32,74 / $48 \pm 5,59$ / uma visão mais positiva); percepção dos docentes (32,71 / $44 \pm 6,23$ / na direção certa); percepção dos resultados acadêmicos (20,22 / $32 \pm 4,09$ / sentimento positivo); percepção do ambiente geral (31,74 / $48 \pm$ 5,69 / atitude positiva); percepção das relações sociais (17,42 / $28 \pm 3,83$ / não é tão ruim). Foram encontrados alguns pontos problemáticos em relação a fatos memorizáveis, metodologia de ensino prévio e cansaço para cursarem a disciplina. A dimensão com mais áreas fortes foi a percepção dos docentes. Houve significância estatística quando os percentuais da média dos escores dos domínios e do Dreem global foram comparados. Conclusão: O ambiente educacional das disciplinas de Semiologia I e II da Faculdade de Medicina da UFG criado pelas inovações metodológicas foi avaliado positivamente pelos discentes participantes, com destaque para o domínio percepção dos docentes. 


\section{KEYWORDS}

- Educational Measurement.

- Students Medical.

- Education medical.

- Teaching.

- Higher Education Institutions.

\section{ABSTRACT}

Introduction: In 2014, the Medical School of the Federal University of Goias (FM-UFG) started its curricular changing process. The disciplines of Semiology I and II started to use active teaching methodologies to fit the curricular reform needs. The objective of this article was to evaluate the FM-UFG students' perception of the Educational Environment in the disciplines of Semiology I and II. Method: This is a descriptive, cross-sectional study encompassing 86 students. The data collection tool was the Dundee Ready Education Environment Measure (DREEM) questionnaire, in its Portuguese version. Results were considered at three levels: individual questions, five domains and global DREEM. Mean values, standard deviation and respective $95 \%$ confidence intervals were calculated. The internal reliability of the DREEM was determined using Cronbach's alpha; the sample normality was determined using the Shapiro-Wilk test; and the comparison between the mean score percentages of the domains and of the global DREEM was performed by ANOVA, followed by Tukey's test. Results: The global DREEM mean was 134.83 / $200 \pm 17.42$, a more positive than negative perception. The five domains showed the following results: Students' Perception of Learning - SPL (32.74 / $48 \pm 5.59$ / a more positive approach); Students' Perception of Teachers - SPT (32.71/ $44 \pm 6.23$ / going in the right direction); Students' Academic Self-Perception - SASP: (20.22 / $32 \pm 4.09$ / feeling more on the positive side); Students' Perception of the Atmosphere - SPA (31.74 / $48 \pm 5.69$ / a more positive attitude); Students' Social Self-Perception SSSP: $(17.42 / 28 \pm 3.83$ / not too bad). Some issues were found in relation to: memorizable facts; previous teaching methodology; and, fatigue to attend the discipline. The SPT was the domain with the largest number of strong areas. Statistical significance was found when comparing the mean score percentages of the domains and the global DREEM. Conclusion: Students positively evaluated the educational environment in the subjects Semiology I and II created by the new active teaching methodologies adopted by the institution, with the SPT domain being highlighted.

Recebido em 24/7/19

Aceito em 14/10/19

\section{INTRODUÇÃO}

A tendência atual no ensino em saúde aponta para a utilização de metodologias ativas centradas no aluno. Nesse contexto, ele é o protagonista do seu processo de formação, transformando o papel do docente de transmissor a facilitador e motivador. Deve ser implementado também um ensino integrado e interdisciplinar, com a inserção do discente desde o início do curso em campos de prática na comunidade. E isso deve estar articulado com a assistência voltada para determinantes biopsicossociais do processo saúde-doença, a fim de inserir a tecnologia da informação no processo de formação ${ }^{1,2}$.

No Brasil, as novas Diretrizes Curriculares Nacionais (DCN) para os cursos de Medicina têm impulsionado mudanças na formação acadêmica, e diversas universidades brasileiras têm passado por processo de transformação nos seus programas de ensino ${ }^{3-5}$. A avaliação dessas mudanças do processo ensino-aprendizagem é fundamental para a implementação e o desenvolvimento das atividades educacionais, pois trata-se de um processo sistemático que documenta e determina a extensão e magnitude do alcance dos objetivos institucionais com subsequente julgamento e tomada de decisão ${ }^{6}$. Desse modo, a análise o torna mais dinâmico, com atualização constante dos objetivos do aprendizado $o^{6,7}$.

Nesse contexto avaliativo de mudanças curriculares, o ambiente educacional é um quesito importante a ser considerado ${ }^{8}$. Em termos de educação médica, definimos o ambiente educacional pelo conjunto de elementos materiais e afetivos que envolvem o graduando, sendo esses fatores ligados à instituição, aos docentes e aos próprios discentes? Ele, portanto, é a manifestação mais significativa do currículo porque envolve tudo o que acontece em um curso de Medicina, inclusive os comportamentos de alunos e professores ${ }^{10}$.

A percepção do discente sobre o ambiente educacional pode influenciar sua satisfação com o curso, sua autopercepção de bem-estar e o seu desempenho acadêmico durante o seu período de formação ${ }^{8}$. Essa análise pode fornecer subsídios importantes para que os elementos positivos sejam mantidos e reforçados, e os aspectos negativos, eliminados ou corrigidos ${ }^{9}$.

Desse modo, o objetivo do estudo foi avaliar a percepção discente sobre o ambiente educacional da disciplina de Semiologia do curso de Medicina.

\section{MÉTODOS}

Delineamento e local do estudo

Trata-se de estudo descritivo, realizado no dia 20 de outubro de 2017, na Faculdade de Medicina da Universidade Federal de Goiás (FM-UFG), em Goiânia, cidade com aproximadamente um milhão de habitantes.

A FM-UFG vem ao longo dos anos propondo mudanças curriculares, e uma nova fase de reforma curricular foi implementada em 2014 para os primeiros quatro anos da graduação com proposta de mudança gradativa e anual. Essas alterações alcançaram maior integração intermodular, inserção precoce do discente nos cenários de práticas e utilização de metodologias ativas de ensino-aprendizagem ${ }^{11}$.

As disciplinas de Semiologia I e II, no primeiro e segundo semestre do curso, respectivamente, visando adequar-se à reforma curricular impulsionada pelas DCN, passaram a trabalhar a parte teórica por meio da metodologia ativa de aprendizado baseado em equipes (team based learning - TBL) ${ }^{12,13}$ e a parte prática pela abordagem de paciente simulado $^{14}$ para alcançar o objetivo principal de capacitar os discentes a coletar e estruturar a anamnese. 
O conteúdo, assim, começou a ser abordado a partir de grandes síndromes, sendo o material disponível em plataforma Moodle aos acadêmicos para estudo prévio. Os docentes passaram a preparar a anamnese completa, abrangendo a síndrome em estudo, e a encaminhar ao Núcleo Livre de Interpretação Teatral Paciente Simulado da Faculdade de Música e Artes Cênicas da UFG para preparo dos atores, a fim de atuarem como paciente simulado.

Participantes

A amostra não probabilística foi composta por acadêmicos do segundo semestre do curso de Medicina da UFG.

Os critérios de inclusão foram os seguintes: 1. os alunos deveriam ter idade superior ou igual a 18 anos, 2. possuir o português como língua nativa, 3. estar devidamente matriculados na disciplina de Semiologia II da FM-UFG no segundo semestre do ano de 2017 e 4. cursar a disciplina de Semiologia I já reestruturada.

Excluíram-se aqueles que 1. estavam ausentes ao momento da abordagem para coleta de dados, 2. preencheram o questionário de maneira incompleta e 3. cursaram Semiologia I previamente à reestruturação.

\section{Coleta de dados}

A coleta de dados ocorreu num único momento, em ambiente reservado na FM-UFG, pela pesquisadora responsável e por quatro auxiliares. Todos os alunos que estavam presentes no dia da coleta foram convidados a participar. Após esclarecimento dos objetivos e métodos do estudo, os discentes, que aceitaram participar, assinaram o Termo de Consentimento Livre e Esclarecido (TCLE).

\section{Instrumento utilizado}

O instrumento Dundee Ready Education Environment Measure (Dreem) foi aplicado a todos os participantes da amostra. Ele foi desenvolvido, originalmente, na língua inglesa em 1997 e teve sua primeira versão em português publicada em $2003^{15,16}$.

Esse instrumento é autoaplicável, de fácil preenchimento e composto por cinco dimensões (percepção da aprendizagem, percepção sobre os docentes, percepção dos resultados acadêmicos, percepção do ambiente geral e percepção das relações sociais) e 50 questões estruturadas em escala Likert com cinco possíveis respostas (0-4), e existem nove questões em que a pontuação ocorre de maneira inversa, pois são constatações de cunho negativo ${ }^{15}$.

Os resultados devem ser considerados em três níveis: questões individuais, cinco dimensões e Dreem global. As respostas obtidas em cada item das cinco dimensões são somadas para cada participante; em seguida, a média desse resultado fornece a pontuação resumida das dimensões. O Dreem global, obtido pela soma das pontuações das dimensões, varia de 0 a 200 pontos, e quanto maior a pontuação, melhor o ambiente educacional ${ }^{15}$. A interpretação das pontuações ocorreu de acordo com proposto por McAleer e Roff ${ }^{17}$.

\section{Desfecho}

O desfecho foi o ambiente educacional das disciplinas de Semiologia I e II, avaliado por meio do questionário Dreem ${ }^{15-17}$.

\section{Variáveis independentes}

As variáveis independentes para a caracterização da amostra foram: idades (em anos vividos) e sexo (feminino ou masculino).

\section{Análise dos dados}

Inicialmente, realizou-se a análise descritiva das variáveis do estudo. As variáveis qualitativas foram apresentadas como frequência absoluta e relativas, enquanto as quantitativas como média e desvio padrão, acompanhadas dos respectivos intervalos de confiança de 95\% (IC 95\%).

A confiabilidade interna do Dreem foi obtida pelo cálculo do alfa de Cronbach, sendo valores $\geq 0,7$ bons indicativos de confiabilidade ${ }^{18}$. Aplicou-se o teste de Shapiro-Wilk para verificar a distribuição dos dados da amostra ${ }^{19}$. A ANOVA e o teste de Tukey foram realizados para avaliar diferença estatística entre os percentuais de escores das médias das cinco dimensões e do Dreem global $^{20}$. A probabilidade de rejeitar a hipótese nula foi de $5 \%$

\section{Aspectos éticos}

A pesquisa inscrita com Certificado de Apresentação para Apreciação Ética (CAAE) no 72207317.6.0000.5078 foi aprovada pelo Comitê de Ética em Pesquisa do Hospital das Clínicas da Universidade Federal de Goiás sob o número de Parecer no 2.245.749. A pesquisa foi realizada segundo as normas brasileiras de pesquisa envolvendo seres humanos ${ }^{21}$.

\section{RESULTADOS}

Dos 111 alunos que se matricularam em Semiologia I, 107 permaneceram matriculados na Semiologia II no dia da coleta dos dados. Destes 90 preencheram o instrumento, todavia quatro de forma incompleta. Logo, a amostra final foi composta por 86 discentes, sendo $58 \%$ do sexo masculino, com média de idade de 19,98 anos (18-32 anos).

Os valores do alfa de Cronbach nas cinco dimensões e no Dreem global se encontram na Tabela 1 . Na avaliação das questões individuais, a maioria apontou para áreas fortes e a melhorar. Foram encontrados alguns pontos problemáticos em relação aos fatos memorizáveis, à metodologia de ensino prévio e ao cansaço para cursarem a disciplina. A dimensão com mais áreas fortes foi a percepção dos docentes.

A avaliação final das percepções dos discentes nas dimensões e no Dreem global e a distribuição dos dados da amostra estão demonstradas na Tabela 2.

Houve significância estatística entre os percentuais de escores das médias da dimensão percepção dos docentes e das demais e do Dreem global, conforme mostra a Tabela 3. A dimensão percepção da aprendizagem apresentou diferença estatística apenas em relação à percepção das relações sociais

\section{Tabela 1}

Alfa de Cronbach por dimensão do Dreem e Dreem global $(n=86)$ DIMENSÃO

ALFA DE CRONBACH

$\begin{array}{cc}\text { Percepção da aprendizagem } & 0,77 \\ \text { Percepção dos docentes } & 0,83 \\ \text { Percepção dos resultados acadêmicos } & 0,68 \\ \text { Perção do ambiente geral } & 0,76 \\ \text { Percepção das relações sociais } & 0,61 \\ \text { Dreem global } & 0,72\end{array}$

Fonte: Elaborada pelos autores

REVISTA BRASILEIRA DE EDUCAÇÃo MÉDICA

3 44 (1) : e012; 2020 


\begin{tabular}{|c|c|c|c|c|c|}
\hline \multicolumn{6}{|c|}{$\begin{array}{c}\text { Tabela } 2 \\
\text { Média, desvio padrão, com respectivos IC de 95\%, interpretação } \\
\text { da pontuação e teste Shapiro-Wilk de normalidade nas respectivas } \\
\text { dimensões do Dreem e Dreem global }(\mathrm{n}=86)\end{array}$} \\
\hline DIMENSÃO & M & DP & IC 95\% & Interpretação & $\mathbf{p}^{*}$ \\
\hline $\begin{array}{l}\text { Percepção da } \\
\text { aprendizagem }\end{array}$ & 32,74 & 5,59 & $31,54-33,94$ & $\begin{array}{l}\text { Uma visão } \\
\text { mais positiva }\end{array}$ & 0,458 \\
\hline $\begin{array}{l}\text { Percepção dos } \\
\text { docentes }\end{array}$ & 32,71 & 6,23 & $31,36-34,04$ & $\begin{array}{l}\text { Na direção } \\
\text { certa }\end{array}$ & 0,007 \\
\hline $\begin{array}{c}\text { Percepção dos } \\
\text { resultados } \\
\text { acadêmicos }\end{array}$ & 20,22 & 4,09 & $19,34-21,09$ & $\begin{array}{l}\text { Sentimento } \\
\text { positivo }\end{array}$ & 0,753 \\
\hline $\begin{array}{l}\text { Percepção do } \\
\text { ambiente geral }\end{array}$ & 31,74 & 5,69 & $30,52-32,96$ & $\begin{array}{l}\text { Atitude } \\
\text { positiva }\end{array}$ & 0,846 \\
\hline $\begin{array}{l}\text { Percepção das } \\
\text { relações sociais }\end{array}$ & 17,42 & 3,83 & $16,59-18,23$ & $\begin{array}{l}\text { Não é tão } \\
\text { ruim }\end{array}$ & 0,515 \\
\hline Dreem global & 134,83 & 17,68 & $130,66-138,24$ & $\begin{array}{l}\text { Mais positivo } \\
\text { que negativo }\end{array}$ & 0,284 \\
\hline
\end{tabular}

M: média; DP: desvio padrão; IC: intervalo de confiança.

*Teste Shapiro-Wilk.

Fonte: Elaborada pelos autores

\section{DISCUSSÃO}

Os discentes avaliaram positivamente o ambiente educacional da disciplina de Semiologia do curso de Medicina da FM-UFG. A dimensão percepção dos docentes foi caracterizada com mais áreas fortes, enquanto a percepção das relações sociais obteve a menor média.

Pode-se fazer um diagnóstico do ambiente educacional das disciplinas com levantamento de áreas fortes e fracas por meio das questões individuais ${ }^{15,17}$. Na percepção da aprendizagem e percepção dos resultados acadêmicos, ratificou-se que a memorização de conteúdo não é algo que agrada aos participantes da amostra.

No contexto das disciplinas de Semiologia, embora o TBL seja uma metodologia ativa de ensino-aprendizagem, os discentes podem se sentir pressionados com a quantidade de objetivos de aprendizagem e material para leitura prévia à aula ${ }^{12}$. Esse deve ser um ponto a ser repensado e trabalhado em conjunto entre discentes e docentes para melhoria da percepção. Uma das críticas a esse método é justamente o fato de o instrutor identificar as necessidades de aprendizagem do estudante, usurpando-lhe a autonomia de explorar determinado conteúdo ${ }^{12,13}$.

Kohoulat, Hayat, Dehghani, Kojuri e Amini ${ }^{22}$ associaram a percepção dos alunos acerca do ambiente educacional com seus resultados cognitivos, comportamentais e emocionais, e concluíram que os estudantes apresentaram experiências mais positivas com o aprendizado quando seus interesses e suas necessidades foram considerados no planejamento do ensino. $\mathrm{O}$ estudo de Guimaraes, Falbo, Menezes e Falbo ${ }^{23}$ sobre o ambiente educacional do internato em pediatria e obstetrícia também encontrou como ponto fraco o ensino que enfatiza fatos memorizáveis, mesmo em se tratando de ambiente de prática. Outras pesquisas também apontaram esse item como problemático ${ }^{24,25}$.

\begin{tabular}{|c|c|c|}
\hline \multicolumn{3}{|c|}{ Dreem global $(\mathrm{n}=86)$} \\
\hline DOMÍNIO & $\begin{array}{c}\text { MÉDIAS PERCENTUAIS } \\
(\%)\end{array}$ & $\mathbf{p}^{*}$ \\
\hline Percepção das relações sociais & 62,21 & a \\
\hline $\begin{array}{c}\text { Percepção dos resultados } \\
\text { acadêmicos }\end{array}$ & 63,19 & $\mathrm{ab}$ \\
\hline Percepção do ambiente geral & 66,13 & $\mathrm{ab}$ \\
\hline Dreem global & 67,23 & $\mathrm{ab}$ \\
\hline Percepção da aprendizagem & 68,22 & $\mathrm{~b}$ \\
\hline Percepção dos docentes & 74,33 & c \\
\hline
\end{tabular}

*ANOVA seguida pelo teste de Tukey; letras diferentes representam $\mathrm{p}<0,05$. Fonte: Elaborada pelos autores

$\mathrm{Na}$ dimensão percepção dos resultados acadêmicos, constataram-se também a dificuldade dos participantes em se adaptar às metodologias inovadoras e certa insegurança para cursar a disciplina. $O$ processo de aprendizagem não deve ser visto como uma transferência de conhecimentos com a memorização de informações. Deve-se partir da análise de situações e da atitude para derivar o conhecimento, abrangendo, entre outras características, conhecimento, capacidade e habilidade de execução, raciocínio, pensamento crítico, postura profissional e ética, relacionamento humano, comportamento, valores, mudança de atitude e até certa independência para a produção do saber $^{26,27}$. Entretanto, a educação básica ainda está muito enraizada na forma de ensino tradicional, sendo um desafio a sua inserção nas metodologias ativas no início da graduação ${ }^{28}$.

$\mathrm{Na}$ percepção das relações sociais, o estudo revelou que é imprescindível levantar os motivos que os tornam tão cansados para aproveitar a disciplina. O núcleo livre de suporte e apoio aos discentes (mentoring) $^{29}$ e o Programa de Acolhimento ao Calouro de Medicina ${ }^{30}$ poderiam ser mais bem divulgados na disciplina para haver maior socialização dos alunos e, consequentemente, amenizar as situações problemáticas supracitadas.

Alguns estudos também indicaram pontuações menores no domínio de relações sociais e a falta de sistema de suporte para alunos estressados e cansados como aspecto problemático, embora o programa de mentoring ou apoio psicopedagógico seja disponibilizado aos discentes ${ }^{23,25,31}$.

A dimensão com mais áreas fortes foi a percepção dos docentes. Houve demonstração de boa percepção pelos discentes com pontuação muito próxima à categoria de excelência. Isso expressa que os docentes conseguem trabalhar bem a nova metodologia proposta pela disciplina, diferentemente do estudo de Kohli e Dhaliwal ${ }^{25}$ que demonstrou muitos pontos problemáticos nesse domínio e os correlacionou com o currículo tradicional.

$\mathrm{Na}$ dimensão percepção do ambiente geral, os discentes apontaram que a disciplina Semiologia Médica proporciona boa experiência e tranquilidade durante as aulas. AI-Hazimi et al..$^{32}$ realizaram estudo em três universidades árabes que adotavam o ensino tradicional e demonstraram muitas áreas problemáticas nessa dimensão. Quando essas instituições foram comparadas 
a uma universidade inglesa com metodologia ativa centrada no estudante, a dimensão atingiu pontuações consideravelmente maiores

Shehnaz, Sreedharan e Gomathi ${ }^{31}$ avaliaram o ambiente educacional quando o ensino era tradicional e depois das mudanças curriculares. As pontuações gerais do Dreem foram 119,18 e 139,96, respectivamente, corroborando a pontuação obtida no nosso estudo, após a inovação da metodologia. AI-Hazimi et al. ${ }^{32}$ compararam uma escola médica inovadora com três tradicionais e a média da pontuação geral foi muito superior e a favor da primeira. Zawawi e Elzubeir ${ }^{33}$ também avaliaram duas escolas médicas, uma com currículo híbrido de aprendizagem baseada em problemas (problem based learning - PBL) e outra tradicional, e a pontuação geral do Dreem da primeira foi superior.

O processo de ensino-aprendizagem não pode permanecer parado ante as transformações. É fundamental que se promovam inovações para que esse processo possa cumprir o seu papel social de formar o profissional que atenda às demandas dessa nova sociedade, o que significa ultrapassar os limites do treinamento técnico ${ }^{34}$. As tendências atuais no ensino em saúde apontam para a utilização de metodologias ativas, em que o aluno é o protagonista do seu próprio processo de formação e o professor se transforma em um facilitador e motivador desse processo, sempre em busca de um ensino integrado e interdisciplinar ${ }^{1,2}$.

Embora o método Dreem seja um instrumento validado para avaliar o ambiente educacional com levantamento de áreas fortes, carentes de melhorias e problemáticas, ele não traz uma avaliação aprofundada da questão ${ }^{15-17}$. Sugere-se complementar o estudo com uma pesquisa qualitativa para melhor compreender os pontos fracos levantados. Devese trabalhar com o universo dos significados, motivos e valores, assim como das aspirações, percepções, crenças e atitudes como parte da realidade social, já que o ser humano não se caracteriza apenas por suas ações, mas também porque é capaz de pensá-las e interpretá-las dentro do contexto vivido ${ }^{35}$.

\section{CONCLUSÃO}

A percepção discente sobre o ambiente educacional das disciplinas de Semiologia Médica é positiva. A dimensão mais bem avaliada é a percepção dos docentes.

Há áreas problemáticas que necessitam de melhoria e áreas fortes que podem ser reforçadas para que as próximas turmas possam avaliar o ambiente educacional das disciplinas como modelo a ser seguido por outras disciplinas da Faculdade de Medicina que também estão em processo de reestruturação.

\section{REFERÊNCIAS}

1. Anastasiou LGC, Alves LP. Processos de ensinagem na universidade: pressupostos para estratégias de trabalho em aula. 9. ed. Joinville: Univille; 2010.

2. Batista N, Batista SH, Goldenberg P, Seiffert O, Sonzogno MC. O enfoque problematizador na formação de profissionais da saúde. Rev Saúde Púb 2005;39(2):231-7.

3. Brasil. Conselho Nacional de Educação. Câmara de Educação Superior. Resolução n 3 , de 20 de junho 2014. Institui Diretrizes Curriculares Nacionais do Curso de Graduação em Medicina. Brasília, DF: CNE/CES, 2014 [acessso em 20 jan 2019].
Disponível em: http://portal.mec.gov.br/index.php?option=com docman \&view = download \&alias $=15874-$ rces003-14\&category slug=junho-2014-pdf\&Itemid=30192.

4. Franco CAGS, Cubas MR, Franco RS. Currículo de medicina e as competências propostas pelas diretrizes curriculares. Rev Bras Educ Med 2014;38(2):221-30.

5. Nogueira MI. As mudanças na educação médica brasileira em perspectiva: reflexões sobre a emergência de um novo estilo de pensamento. Rev Bras Educ Med 2009;33(2):262-70.

6. Goldie J. AMEE Education Guide $\mathrm{n}^{\circ}$ 29: evaluating educational programmes. Med Teach 2006; 28(3):210-24.

7. Al-Khathami AD. Evaluation of saudi family medicine training program: the application of CIPP evaluation format. Med Teach 2012;34:S81-9.

8. Miles S, Swift L, Leinster SJ. The Dundee Ready Education Environment Measure (DREEM): a review of its adoption and use. Med Teach 2012;34(9):620-34.

9. Troncon, LEA. Ambiente educacional. Medicina (Ribeirão Preto). 2014;47(3):264-71.

10. Geen, JM. AMEE Medical Education Guide $n^{\circ} 23$ (PART 1): Curriculum, environment, climate, quality and change in medical education - a unifying perspective. Med Teac 2001;23(4):337- 44.

11. Universidade Federal de Goiás. Faculdade de Medicina Projeto político pedagógico. Goiânia: UFG; 2017 [acesso em 20 jan 2019]. Disponível em: https://www.medicina.ufg.br/up/148/o/PPC-2017. pdf?1505222188.

12. Bollela VR, Senger MH, Tourinho FSV, Amaral E. Aprendizagem baseada em equipes: da teoria à prática. Medicina (Ribeirão Preto). 2014;47(3):293-300.

13. Parmelee DX, Michaelsen LK. Twelve tips for doing effective TeamBased Learning (TBL). Med Teach 2010;32(2):118-22.

14. Joyner B, Young L. Teaching medical students using role play: twelve tips for successful role plays. Med Teach 2006;28(3):225-9.

15. Roff S, McAleer S, Harden RM, Al-Qahtani M, Ahmed AU, Deza H et al. Development and validation of the Dundee Ready Education Environment Measure (DREEM). Med Teach1997;19(4): 295-9.

16. Vieira JE, Nunes MPT, Martins MA. (2003) Directing student response to early patient contact by questionary. Med Educ 2003;37(2):119-25.

17. McAleer S, Roff S. A practical guide to using the Dundee Ready Education Environment Measure (DREEM). In Curriculum, Environment, Climate, Quality and Change in Medical Education: a Unifying Perspective. AMEE Education Guide no 23. Dundee: Association for Medical Education in Europe Edited by Genn JM 2001:29-33.

18. Gliem JA, Gliem RR. Calculating, interpreting, and reporting Cronbach's alpha reliability coefficient for Likert-type scales. Midwest Res to Pract Conf Adult Contin Community Educ 2003:82-8.

19. Chan YH. Biostatistics 101: data presentation. Singapore Med J 2003;44(6):280-5.

20. Chan YH. Biostatistics 102: quantitative data: parametric \& non parametric tests. Singapore Med J 2003;44(8):391-6.

21. Brasil. Conselho Nacional de Saúde. Resolução $n^{\circ} 466$, de 12 de dezembro de 2012. Aprova as diretrizes e normas regulamentadoras

$5 \mid$\begin{tabular}{l|l} 
REVISTA BRASILEIRA DE EDUCAÇÃO MÉDICA \\
\hline 44 (1) : e012; 2020
\end{tabular} 
de pesquisa envolvendo seres humanos. Brasília, DF: CNS; 2012 [acesso em 20 ago 2017]. Disponível em: http://conselho.saude.gov. br/resolucoes/2012/Reso466.pdf.

22. Kohoulat N, Hayat AA, Dehghani MR, Kojuri J, Amini M. Medical students' academic emotions: the role of perceived learning environment. J Adv Med Educ Prof 2017;5(2):78-83.

23. Guimaraes AC, Falbo GH, Menezes T, Falbo A. Percepção do estudante de medicina acerca do ambiente educacional utilizando o Dreem. Rev Bras Educ Méd 2015;39(4):517-26.

24. Patil AA, Chaudhari VL. Student's perception of the educational environment in medical college: a study based on DREEM questionnaire. Korean J Med Educ 2016;28(3):281-8.

25. Kohli V, Dhaliwal U. Medical students' perception of the educational environment in a medicai college in lndia: a cross-sectional study using the Dundee Ready Education Environment questionnaire. J Educ Eval Health Prof 2013;10(5).

26. Norcini JJ, McKinley DW. Assessment methods in medical education. Teach Teach Educ 2007; 23(3):239-50.

27. lrby DM, Wilkerson L. Educational innovation in academic medicine and environmental trends. J Gen lntern Med 2003;18(5):370-6.

28. Paiva MRF, Parente JRF, Brandão IR, Queiroz AHB. Metodologias ativas de ensino-aprendizagem: revisão integrativa. Sanare 2016;15(2):145-53.

29. Pereira MAD. Tutoria (mentoring) na Faculdade de Medicina da UFG. Universidade Federal de Goiás; 2017 [acesso em 20 jan 2019]. Disponível em: http:// www.medicina.ufg.br/p/21108-tutoria-2015projeto-inicial.

30. Programa de Acolhimento ao Calouro de Medicina (PAC-Med): 2018-2019. Sistema Integrado de Gestão de Atividades Acadêmicas da Universidade Federal de Goiás; 2018 [acesso em 20 jan 2019]. Disponível em: http:// sigaa.sistemas.ufg.br/sigaa/public/extensao/ consulta_extensao.jsf.

31. Shehnaz SI, Sreedharan J, Gomathi KG. Does curricular change improve faculty perceptions of student experiences with the educational environment? A preliminary study in an institution undergoing curricular change. J Educ Eval Health Prof 2014;11(7).

32. AI-Hazimi A, Zaini R, AI-Hyiani A, Hassan N, Gunaid A., Ponnamperuma $\mathrm{G}$ et al. Educational environment in traditional and innovative medical schools: a study in four undergraduate medical schools. Educ Health 2004;17(2):192-203.

33. Zawawi AH, Elzubeir M. Using DREEM to compare graduating students perceptions of learning environments at medical schools adopting contrasting educational strategies. Med Teach 2012; 34:S25-31.
34. Pagliosa FL, Da Ros MA. O relatório Flexner: para o bem e para o mal. Rev Bras Educ Méd 2008;32(4):492-9.

35. Minayo MCS, Gomes SFD. Pesquisa social: teoria, método e criatividade. 29. ed. Petrópolis: Vozes; 2010.

\section{CONTRIBUIÇÃO DOS AUTORES}

Desenho e concepção do projeto e coleta de dados: Sílvia Cristina Marques Nunes Pricinote, Artur Lorenzo Sena Gomes, Alberto Monteiro Filho, Bruno Leonardo Wadson Silva e Reinaldo Elias de Souza Junior.

Análise estatística: Sílvia Cristina Marques Nunes Pricinote, Artur Lorenzo Sena Gomes, Alberto Monteiro Filho, Bruno Leonardo Wadson Silva, Reinaldo Elias de Souza Junior e Marcos Rassi Fernandes.

Análise e interpretação dos dados: Sílvia Cristina Marques Nunes Pricinote, Marcos Rassi Fernandes, Maria do Rosário Ferraz Roberti e Denise Milioli Ferreira.

Elaboração do manuscrito: Sílvia Cristina Marques Nunes Pricinote, Artur Lorenzo Sena Gomes, Alberto Monteiro Filho, Bruno Leonardo Wadson Silva, Reinaldo Elias de Souza Junior, Marcos Rassi Fernandes, Maria do Rosário Ferraz Roberti e Denise Milioli Ferreira.

Revisão crítica do manuscrito quanto a conteúdo intelectual importante: Sílvia Cristina Marques Nunes Pricinote, Marcos Rassi Fernandes, Maria do Rosário Ferraz Roberti e Denise Milioli Ferreira.

Suporte administrativo, técnico ou material: Sílvia Cristina Marques Nunes Pricinote, Artur Lorenzo Sena Gomes, Alberto Monteiro Filho, Bruno Leonardo Wadson Silva e Reinaldo Elias de Souza Junior.

Supervisão do estudo: Sílvia Cristina Marques Nunes Pricinote, Marcos Rassi Fernandes, Maria do Rosário Ferraz Roberti e Denise Milioli Ferreira.

\section{CONFLITO DE INTERESSES}

Os autores declaram não haver conflitos de interesse neste estudo.

\section{ENDEREÇO PARA CORRESPONDÊNCIA}

Sílvia Cristina Marques Nunes Pricinote

Rua 235 com $1^{\text {a }}$ avenida, s/n, Universitário, Goiânia, Goiás, CEP 74605-020.

E-mail: dra.silvia.pricinote@gmail.com 